\title{
De novo prions
}

Federico Benetti ${ }^{1,2}$, Michael D Geschwind ${ }^{3}$ and Giuseppe Legname $e^{1,2,4 *}$

\author{
Addresses: ${ }^{1}$ Laboratory of Prion Biology, Neurobiology Sector, Scuola Internazionale Superiore di Studi Avanzati - International School of \\ Advanced Studies (SISSA-ISAS), via Bonomea 265, 34136 Trieste, Italy; ${ }^{2}$ Italian Institute of Technology, SISSA-ISAS Unit, via Bonomea 265,34136 \\ Trieste, Italy; ${ }^{3}$ Memory and Aging Center, Department of Neurology, University of California, San Francisco, Medical Center, Box 1207 , San \\ Francisco, CA 94117, USA; ${ }^{4}$ ELETTRA Laboratory, Sincrotrone Trieste S.C.p.A., S.S. 14 Km 163.5, 34012 Basovizza, Trieste, Italy \\ *Corresponding author: Giuseppe Legname (legname@sissa.it) \\ FI000 Biology Reports 2010, 2:46 (doi:10.34I0/B2-46)
}

The electronic version of this article is the complete one and can be found at: http://fl000.com/reports/biology/content/2/46

\begin{abstract}
Prions are responsible for a heterogeneous group of fatal neurodegenerative diseases. They occur in three forms - sporadic, genetic, or acquired - and involve non-covalent post-translational modifications of the cellular prion protein $\left(\mathrm{PrP}^{\mathrm{C}}\right)$. Prions $\left(\mathrm{Pr}^{\mathrm{Sc}}\right)$ are characterized by their infectious properties and intrinsic ability to act as a template, converting the normal, physiological $\mathrm{PrP}^{\mathrm{C}}$ into the pathological form, PrPSc. The 'protein-only' hypothesis, postulated by Stanley B Prusiner, implies that the generation of de novo prions is possible. Exciting recent work, in vivo and in vitro, has further strengthened this postulate.
\end{abstract}

\section{Introduction and context}

Prions, proteinaceous infectious particles, or $\operatorname{PrP}^{\mathrm{Sc}}$, are responsible for a heterogeneous group of fatal neurodegenerative diseases [1]. Prion diseases manifest as sporadic, genetic, or acquired disorders, involving noncovalent, post-translational modifications of the normal cellular prion protein $\left(\mathrm{PrP}^{\mathrm{C}}\right.$, where the superscript $\mathrm{C}$ stands for cellular) into $\mathrm{PrP}^{\mathrm{Sc}}$ (where the superscript $\mathrm{Sc}$ stands for scrapie, the prion disease that affects sheep) [2]. Prions are characterized by their infectious properties and by the intrinsic ability of their secondary, tertiary, and quaternary structures to act as a template and convert the normal physiological $\mathrm{PrP}^{\mathrm{C}}$ into the pathological, diseasecausing form, $\operatorname{PrP}^{\mathrm{SC}}[3]$.

\section{Major recent advances}

\section{In vitro support for de novo prions}

The 'protein-only' hypothesis for prion propagation implies that it should be possible to generate prions in vitro from highly purified recombinant $\operatorname{PrP}$ (recPrP). Several attempts have been made to confirm this hypothesis, but the resulting prions had little or no infectivity, as well as minimal or no protease digestion resistance - two elements necessary to prove the 'protein- only' hypothesis $[4,5]$. In recent years, the most striking work supporting the 'protein-only' postulate has shown that pure recPrP can be converted in vitro into a structural form similar to prions [6-9]. When $\mathrm{PrP}^{\mathrm{Sc}}$ is treated with proteinase $\mathrm{K}(\mathrm{PK})$ and further processed, the $\mathrm{N}$-terminal region is removed, resulting in a PK-resistant core with an apparent molecular mass of 27-30 kDa, called PrP2730 [10]. Legname et al. [6] produced truncated recombinant mouse PrP (MoPrP), corresponding to PrP27-30, and then converted this into amyloid fibrils. Amyloid fibrils were intracerebrally inoculated into transgenic (Tg) mice over-expressing the N-terminally truncated form of MoPrP, MoPrP(89-231). These mice, called Tg9949, express $\operatorname{MoPrP}(89-231)$ at levels 16 times higher than the normal $\operatorname{PrP}^{\mathrm{C}}$ expressed in wild-type mice [11]. These inoculated fibrils caused neurological defects between 380 and 660 days post-inoculation (DPI), depending on the biochemical protocol applied [6]. Brain homogenates of mice inoculated with the amyloid preparations exhibited the PK-resistant form of $\mathrm{PrP}^{\mathrm{Sc}}$. Serial transmission of these brain homogenates caused disease in transgenic and wild-type mice. Thus, the synthetic prions generated using truncated recombinant MoPrP demonstrated that PrP is necessary and sufficient 
for infectivity [6]. This seminal work has further contributed to our understanding of the biochemical and biophysical characteristics of prions, indicating the existence of an inverse correlation between the stability and infectivity of prion isolates [8]. Colby and colleagues [9] took this work a step further; they produced an array of $\operatorname{PrP}$ amyloids with different conformational stabilities and found that the inverse relationship between stability and incubation time was still valid. The most stable prion strains exhibited the longest incubation times, whereas more labile amyloids revealed shorter incubation times [9]. More recently, Colby and colleagues [12] isolated protease-sensitive synthetic prion $\left(\mathrm{sPrP}^{\mathrm{Sc}}\right)$. Often, $\operatorname{PrP}^{\mathrm{Sc}}$ is protease-resistant but $\operatorname{sPrP}^{\mathrm{Sc}}$ has been isolated in humans and other animals. Colby et al. reported that $\mathrm{sPrP}^{\mathrm{Sc}}$ was generated in vitro during polymerization of recPrP into amyloid fibers. In 22 independent experiments, recPrP amyloid preparations, but not recPrP monomers or oligomers, transmitted disease to Tg9949 mice $(\mathrm{n}=164)$. In addition, they inoculated three synthetic prion isolates into Tg4053 mice that overexpress full-length MoPrP. Tg4053 mice are not prone to developing spontaneous neurological dysfunction [13]. The resulting synthetic prion isolates caused disease in 600-750 DPI in Tg4053 mice, which exhibited $\mathrm{sPrP}^{\mathrm{Sc}}$. These novel synthetic prions demonstrate that conformational changes in wildtype PrP can produce prions composed exclusively of $\operatorname{sPrP}^{\mathrm{Sc}}[12]$.

More recently, Makarava et al. [14] showed additional experimental evidence that synthetic prions can be generated when amyloid fibrils produced from fulllength recombinant Syrian hamster ( $\mathrm{SHa}$ ) PrP were intracerebrally inoculated in SHa animals.

Castilla and colleagues generated infectious scrapie prions [15] using the protein misfolding cyclic amplification (PMCA) in vitro system [16]. PMCA is a procedure involving cyclic amplification of PrP misfolding that allows rapid conversion of large excess $\mathrm{PrP}^{\mathrm{C}}$ into a protease-resistant, prion-like form in the presence of low quantities of $\mathrm{PrP}^{\mathrm{SC}}$ template [16]. In the experiment of Castilla et al., 263K-infected (SHa-adapted scrapie form of prion) SHa brain homogenates were subjected to 20 PMCA cycles in the presence of $\operatorname{PrP}^{\mathrm{C}}$. Although no molecules of the original scrapie brain homogenate were present in samples, biochemical and structural properties of the in vitro-amplified samples still revealed the same features of $263 \mathrm{~K}$ prions. In particular, the amplified sample was PK-resistant, highly insoluble in nondenaturant detergent (such as $10 \%$ sarkosyl), and enriched in $\beta$-sheet content. The PK-resistant-inoculated animals died at around 170 DPI showing the typical signs of scrapie. To verify the PK-resistant in vitrogenerated stability over time, serial transmissions were carried out; animals inoculated with this material exhibited neurological dysfunction and died after a mean of 136.5 DPI.

To investigate the mechanism of prion formation, Deleault et al. [17] conducted a series of experiments using the PMCA in vitro system in the presence of co-purified lipids and polyanions. They generated $\operatorname{PrP}^{\mathrm{Sc}}$ de novo from the simple combination of $\mathrm{PrP}^{\mathrm{C}}$, copurified lipids, and simple polyanions $[17,18]$, although some questions were raised of whether generation of de novo prions from standard PMCA methodology might be due to cross-contamination from existing prions [4].

The PMCA in vitro system has also been used to propagate prion strains [19-21]. Numerous PMCA amplifications were carried out using, as a seed, different mouse-adapted (RML [Rocky Mountain Laboratory], ME7, 139A and 79A), human (vCJD [variant Creutzfeldt-Jakob disease]), bovine (301C), SHa (263K), and cervid (CWD [chronic wasting disease]) prion strains. Biochemical analyses - PK digestion, glycosylation ratio, and electrophoresis mobility - of end products revealed the same strain features as the original seeds, suggesting that strains are dependent on the properties of $\mathrm{PrP}^{\mathrm{Sc}}$ [19-21]. PMCA was also employed with prions from various species in combination with transgenic mice to study, and even overcome, the prion species barrier $[19,21]$. De novo in vitro generation of $\mathrm{PrP}^{\mathrm{Sc}}$ from $\operatorname{PrP}^{\mathrm{C}}$ and poly(A)RNA using the PMCA system has opened up new ways to investigate the molecular mechanisms underlying the spontaneous misfolding of the PrP and its propagation. To convert de novo $\operatorname{PrP}^{\mathrm{C}}$ from healthy SHa and mouse brain homogenates into its infectious isoform, Barria and colleagues [4] modified the PMCA conditions, increasing the number of PMCA cycles. Animals inoculated with this PK-resistant PrP showed abnormalities after an average incubation period of 112.6 days and revealed spongiform degeneration, astroglyosis, and PrP accumulation. Even more recently, Wang et al. [22] employed PMCA under standard conditions (cycles) and produced prions from bacterially expressed recombinant $\operatorname{MoPrP}(23-230)$ in the presence of the synthetic anionic phospholipid 1-palmitoyl-2oleoylphosphatidylglycerol and RNA (as a polyanion). These prions were protease-resistant. When injected into wild-type mice, this new aggregated and pathogenic PrP isoform induced neurological signs after about $130 \mathrm{DPI}$, with the terminal phase occurring around 150 DPI [22]. Nevertheless, upon second passage the newly discovered prion isolate did not lead to abbreviation or stabilization 
of incubation times in mice, casting doubts about the identification of novel synthetic prions.

\section{In vivo de novo prions}

The existence of human genetic prion diseases, in which patients with mutations in the open-reading frame of the human PrP gene, PRNP (PRioN Protein), spontaneously develop prion disease (transmissible in most forms), strongly supports the 'protein-only' hypothesis $[23,24]$. Additionally, many transgenic mice with mutations homologous to human prion diseases also develop spontaneous disease that is often transmissible [25]. Transgenic mice carrying mutations and over-expressing PrP mutants spontaneously develop neurological symptoms similar to those of prion disorders, but their brain homogenates are infectious only for mice carrying the same mutation, and not for wild-type mice $[5,26,27]$. Therefore, it has been argued in the prion field as to whether this represents true de novo prion generation, or merely an acceleration of disease [28].

Recently, Sigurdson and coworkers [29] developed a transgenic mouse over-expressing two novel mutations in the MoPrP gene, Prnp; these change two amino acids into those normally present in the elk PrP primary sequence that confer a rigid structure to the $\beta 2-\alpha 2$ loop in the protein. Although they created this transgenic mouse to study the role of the rigid-loop structure, surprisingly they found that these mice, called transgenic rigid loop (RL)-PrP mice, developed spontaneous prion disease at variable time points, with a 50\% incidence by 364 DPI. Sick RL-PrP mice showed ataxia, weight loss, lethargy, kyphosis, and hind limb paralysis, while their brains showed PrP deposits in the stratum lacunosum-moleculare of the hippocampus, within the corpus callosum, and in the cingulum. Due to a transmission barrier, RL-PrP mice inoculated with wildtype prion strains exhibited prolonged incubation time before developing prion disease. Therefore, the authors did not attempt to transmit transgenic RL-PrP prions directly to wild-type mice; rather, they stabilized the prion isolate from RL-PrP mice by passaging it in mice overexpressing wild-type $\operatorname{PrP}$, called Tga20 mice [30]. Tga20 mice inoculated with brain homogenates from sick RL-PrP mice developed neurologic signs by 481 DPI. Serial passages into Tga20 mice of brain homogenates from RL-PrP-infected Tga20 mice caused similar symptoms with a shorter incubation period. Moreover, both PK resistance and conformational stability increased after each passage. After serial passaging in Tga20 mice, these de novo generated prions were transmissible to wild-type mice [29]. This is the first time that prions generated spontaneously in mice models are able to infect mice carrying a wild-type sequence.

\section{Future directions}

In order to define a prion, one needs to fulfill four criteria: transmissibility into recipient animals, neuropathological features (e.g., gliosis, vacuolation, immunopositive PrP deposits) [31], biochemical features, such as PK resistance [32], and stability (defined by resistance to denaturation with chaotropic agents, such as guadinium $\mathrm{HCl}$ ) [33]. The strain of a prion is determined by these characteristics. Now, we can design synthetic prions with defined biophysical characteristics and predict their infectivity [9]. A major frontier in prion research is to determine the precise structural properties of the prion at the molecular level, such as differences in secondary, tertiary, and quaternary structures among different strains that confer defined prion strain characteristics.

\section{Abbreviations}

DPI, days post-inoculation; MoPrP, mouse PrP; PK, proteinase K; PMCA, protein misfolding cyclic amplification; PrP, prion protein; $\operatorname{PrP}^{\mathrm{C}}$, 'cellular' $\operatorname{PrP}$ or normal form of PrP; $\operatorname{PrP}^{\mathrm{Sc}}$, 'scrapie' PrP or pathological form of PrP; recPrP, recombinant PrP; RL, rigid loop; SHa, Syrian hamster; $\mathrm{sPrP}^{\mathrm{Sc}}$, sensitive $\mathrm{PrP}^{\mathrm{Sc}} ; \mathrm{Tg}$, transgenic.

\section{Competing interests}

The authors declare that they have no competing interests.

\section{Acknowledgments}

MDG was funded by the Michael J Homer Family fund and the National Institutes of Health National Institute on Aging (NIH/NIA) K23 AG021989 and R01AG031189. GL has received funding from the European Community's Seventh Framework Programme (FP7/ 2007-2013) under grant agreement number 222887 the PRIORITY project.

\section{References}

I. Prusiner SB: Novel proteinaceous infectious particles cause scrapie. Science 1982, 2 16:136-44.

2. Prusiner SB: Prions. Proc Natl Acad Sci U S A 1998, 95:13363-83.

3. Supattapone S: Prion protein conversion in vitro. J Mol Med 2004, 82:348-56.

4. Barria MA, Mukherjee A, Gonzalez-Romero D, Morales R, Soto C: De novo generation of infectious prions in vitro produces a new disease phenotype. PLoS Pathog 2009, 5:el00042I.

5. Hsiao KK, Groth D, Scott M, Yang SL, Serban H, Rapp D, Foster D, Torchia M, Dearmond S], Prusiner SB: Serial transmission in rodents of neurodegeneration from transgenic mice expressing mutant prion protein. Proc Natl Acad Sci U S A 1994 , 91:9126-30.

6. Legname G, Baskakov IV, Nguyen HO, Riesner D, Cohen FE, DeArmond SJ, Prusiner SB: Synthetic mammalian prions. Science 2004, 305:673-6.

FI000 Factor 9.0 Exceptional

Evaluated by Joachim Messing 06 Aug 2004 
7. Legname G, Nguyen HO, Baskakov IV, Cohen FE, Dearmond SJ, Prusiner SB: Strain-specified characteristics of mouse synthetic prions. Proc Natl Acad Sci U S A 2005, 102:2168-73.

8. Legname G, Nguyen HO, Peretz D, Cohen FE, DeArmond SJ, Prusiner SB: Continuum of prion protein structures enciphers a multitude of prion isolate-specified phenotypes. Proc Natl Acad Sci U S A 2006, 103:19105-10.

9. Colby DW, Giles K, Legname G, Wille H, Baskakov IV, DeArmond SJ, Prusiner SB: Design and construction of diverse mammalian prion strains. Proc Natl Acad Sci U S A 2009, 106:20417-22.

10. Prusiner SB, Groth DF, Bolton DC, Kent SB, Hood LE: Purification and structural studies of a major scrapie prion protein. Cell 1984, 38: 127-34.

II. Supattapone S, Muramoto T, Legname G, Mehlhorn I, Cohen FE, DeArmond SJ, Prusiner SB, Scott MR: Identification of two prion protein regions that modify scrapie incubation time. $J$ Virol 200I, 75:1408-13.

12. Colby DW, Wain R, Baskakov IV, Legname G, Palmer CG, Nguyen HO, Lemus A, Cohen FE, DeArmond SJ, Prusiner SB: Protease-sensitive synthetic prions. PLoS Pathog 2010, 6: el000736.

13. Telling GC, Haga T, Torchia M, Tremblay P, DeArmond SJ, Prusiner SB: Interactions between wild-type and mutant prion proteins modulate neurodegeneration in transgenic mice. Genes Dev 1996, 10:1736-50.

14. Makarava N, Kovacs GG, Bocharova O, Savtchenko R, Alexeeva I, Budka H, Rohwer RG, Baskakov IV: Recombinant prion protein induces a new transmissible prion disease in wild-type animals. Acta Neuropathol 2010, I 19:177-87.

15. Castilla J, Saá $P$, Hetz C, Soto C: In vitro generation of infectious scrapie prions. Cell 2005, I2 I:195-206.

FI000 Factor 8.2 Exceptional

Evaluated by David P Wolfer 03 May 2005, Matthias Maiwald 06 Jun 2005, Joe Heitman II Aug 2005

16. Saborio GP, Permanne B, Soto C: Sensitive detection of pathological prion protein by cyclic amplification of protein misfolding. Nature 200I, 4I I:8I0-3.

17. Deleault NR, Geoghegan JC, Nishina K, Kascsak R, Williamson RA, Supattapone S: Protease-resistant prion protein amplification reconstituted with partially purified substrates and synthetic polyanions. J Biol Chem 2005, 280:26873-9.

18. Deleault NR, Harris BT, Rees JR, Supattapone S: Formation of native prions from minimal components in vitro. Proc Natl Acad Sci U S A 2007, 104:974I-6.

19. Castilla J, Morales R, Saá P, Barria M, Gambetti P, Soto C: Cell-free propagation of prion strains. EMBO J 2008, 27:2557-66.

20. Castilla J, Gonzalez-Romero D, Saá P, Morales R, De Castro J, Soto C: Crossing the species barrier by $\operatorname{PrP}(\mathrm{Sc})$ replication in vitro generates unique infectious prions. Cell 2008, 134:757-68.

FI000 Factor 3.2 Recommended

Evaluated by Jose Valpuesta 16 Oct 2008, Art Horwich 20 Oct 2008
2I. Green KM, Castilla J, Seward TS, Napier DL, Jewell JE, Soto C, Telling GC: Accelerated high fidelity prion amplification within and across prion species barriers. PLoS Pathog 2008, 4:el 000139.

22. Wang $F$, Wang $X$, Yuan CG, Ma J: Generating a prion with bacterially expressed recombinant prion protein. Science 2010 , 327: $1132-5$.

FI000 Factor 6.0 Must Read Evaluated by David Lynn 13 Apr 2010

23. Kong Q, Surewicz WK, Petersen RB, Zou W, Chen SG, Gambetti P. Inherited prion diseases. In Prion Biology and Diseases. Edited by Prusiner SB. New York: Cold Spring Harbor Laboratory Press; 2004: 673-775.

24. Mead S: Prion disease genetics. Eur J Hum Genet 2006, 14:273-81.

25. Telling GC: Transgenic mouse models of prion diseases. Methods Mol Biol 2008, 459:249-63.

26. Dossena S, Imeri L, Mangieri M, Garofoli A, Ferrari L, Senatore A, Restelli E, Balducci C, Fiordaliso F, Salio M, Bianchi S, Fioriti L, Morbin M, Pincherle A, Marcon G, Villani F, Carli M, Tagliavini F, Forloni G, Chiesa R: Mutant prion protein expression causes motor and memory deficits and abnormal sleep patterns in a transgenic mouse model. Neuron 2008, 60:598-609.

27. Jackson WS, Borkowski AW, Faas H, Steele AD, King OD, Watson N, Jasanoff $A$, Lindquist $S$ : Spontaneous generation of prion infectivity in fatal familial insomnia knockin mice. Neuron 2009, 63:438-50.

28. Nazor KE, Kuhn F, Seward T, Green M, Zwald D, Pürro M, Schmid J, Biffiger K, Power AM, Oesch B, Raeber AJ, Telling GC: Immunodetection of disease-associated mutant $\operatorname{PrP}$, which accelerates disease in GSS transgenic mice. EMBO J 2005, 24:2472-80.

29. Sigurdson CJ, Nilsson KP, Hornemann S, Heikenwalder M, Manco G, Schwarz P, Ott D, Rülicke T, Liberski PP, Julius C, Falsig J, Stitz L, Wüthrich K, Aguzzi A: De novo generation of a transmissible spongiform encephalopathy by mouse transgenesis. Proc Natl Acad Sci U S A 2009, 106:304-9.

FI000 Factor 9.0 Exceptional

Evaluated by Samuel Gandy 04 Feb 2009

30. Fischer $M$, Rülicke $T$, Raeber $A$, Sailer A, Moser $M$, Oesch B, Brandner S, Aguzzi A, Weissmann C: Prion protein (PrP) with amino-proximal deletions restoring susceptibility of PrP knockout mice to scrapie. EMBO J 1996, 15:1255-64.

31. Kretzschmar HA, Ironside JW, DeArmond SJ, Tateishi J: Diagnostic criteria for sporadic Creutzfeldt-Jakob disease. Arch Neurol 1996, 53:913-20.

32. Prusiner SB: Prions. Sci Am 1984, 25 I:50-9.

33. Peretz D, Williamson RA, Legname G, Matsunaga $Y$, Vergara J, Burton DR, DeArmond SJ, Prusiner SB, Scott MR: A change in the conformation of prions accompanies the emergence of a new prion strain. Neuron 2002, 34:92I-32. 